\title{
BMJ Open Study protocol for a randomised controlled trial of meniscal surgery compared with exercise and patient education for treatment of meniscal tears in young adults
}

\author{
Søren Thorgaard Skou, ${ }^{1,2}$ Martin Lind, ${ }^{3}$ Per Hölmich, ${ }^{4}$ Hans Peter Jensen, ${ }^{5}$ \\ Carsten Jensen, ${ }^{6,7}$ Muhammad Afzal, ${ }^{8}$ Uffe Jørgensen, ${ }^{9}$ Jonas Bloch Thorlund ${ }^{1}$
}

To cite: Skou ST, Lind M, Hölmich P, et al. Study protocol for a randomised controlled trial of meniscal surgery compared with exercise and patient education for treatment of meniscal tears in young adults. BMJ Open 2017;7:e017436. doi:10.1136/ bmjopen-2017-017436

- Prepublication history and additional material are available online. To view, these files please visit the journal (http:// dx.doi.org/10.1136/bmjopen2017-017436).

Received 22 April 2017 Revised 24 June 2017 Accepted 11 July 2017

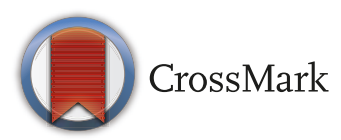

For numbered affiliations see end of article.

Correspondence to Dr Søren Thorgaard Skou; stskou@health.sdu.dk

\section{ABSTRACT}

Introduction Arthroscopic surgery is a very common orthopaedic procedure. While several trials have investigated the effect of knee arthroscopy for middleaged and older patients with meniscal tears, there is a paucity of trials comparing meniscal surgery with nonsurgical treatment for younger adults. The aim of this randomised controlled trial (RCT) is to investigate if early arthroscopic surgery is superior to exercise therapy and education, with the option of later surgery if needed, in improving pain, function and quality of life in younger adults with meniscal tears.

Methods and analysis This is a protocol for a multicentre, parallel-group RCT conducted at six hospitals across all five healthcare regions in Denmark. 140 patients aged 18-40 years with a clinical history and symptoms consistent with a meniscal tear, verified on MRl, found eligible for meniscal surgery by an orthopaedic surgeon will be randomly allocated to one of two groups (1:1 ratio). Participants randomised to surgery will undergo either arthroscopic partial meniscectomy or meniscal repair followed by standard postsurgical care, while participants allocated to exercise and education will undergo a 12-week individualised, supervised neuromuscular and strengthening exercise programme and patient education. The primary outcome will be difference in change from baseline to 12 months in the mean score on four Knee Injury and Osteoarthritis Outcome Score subscales, covering pain, symptoms, function in sports and recreation and quality of life (Knee Injury and Osteoarthritis Outcome Score $\left(\mathrm{KOOS}_{4}\right)$ ) supported by the individual subscale scores allowing clinical interpretation. Alongside, the RCT an observational cohort will follow patients aged 18-40 years with clinical suspicion of a meniscal tear, but not fully eligible or declining to participate in the trial.

Ethics and dissemination Results will be presented in peer-reviewed journals and at international conferences. This study is approved by the Regional Committees on Health Research Ethics for Southern Denmark. Registration details ClinicalTrials.gov (NCT02995551).

\section{BACKGROUND}

Kneearthroscopyisaverycommonlyperformed orthopaedic procedure. ${ }^{12}$ According to

\section{Strengths and limitations of this study}

- This study addresses an important evidence gap regarding the optimal treatment strategy of meniscal tears in young adults.

- The multicentre design including patients from all healthcare regions of Denmark comparing two feasible and clinically relevant treatment strategies will support external validity and implementation.

- Owing to the type of interventions, blinding of the patients and treatment providers is not possible.

the previous reports, around 1 million procedures are performed annually in the $\mathrm{USA}^{3}$ and 150000 procedures in the $\mathrm{UK},{ }^{4}$ with most procedures involving meniscal tears. ${ }^{34}$

Systematic reviews and meta-analyses of randomised trials show no better effect of arthroscopic partial meniscectomy (APM) for middle-aged and older patients with degenerative meniscal tears compared with placebo surgery or in addition to exercise. ${ }^{56}$ In addition, meniscal surgery is associated with risk of adverse events. ${ }^{5}$ A recent randomised controlled trial (RCT) confirmed that exercise therapy is a valid treatment option for middle-aged patients with degenerative meniscal tears as no difference was observed in patient-reported outcomes, when comparing APM surgery with exercise therapy head-tohead. ${ }^{7}$ However, no RCTs have compared arthroscopic meniscal surgery for patients aged 40 years or younger with non-surgical treatments.

In contrast to middle-aged and older people with degenerative meniscal tears most tears in younger adults are of traumatic origin from a work-related or sports-related trauma. ${ }^{9}$ Symptoms associated with meniscal tears are considered to be resolved with 


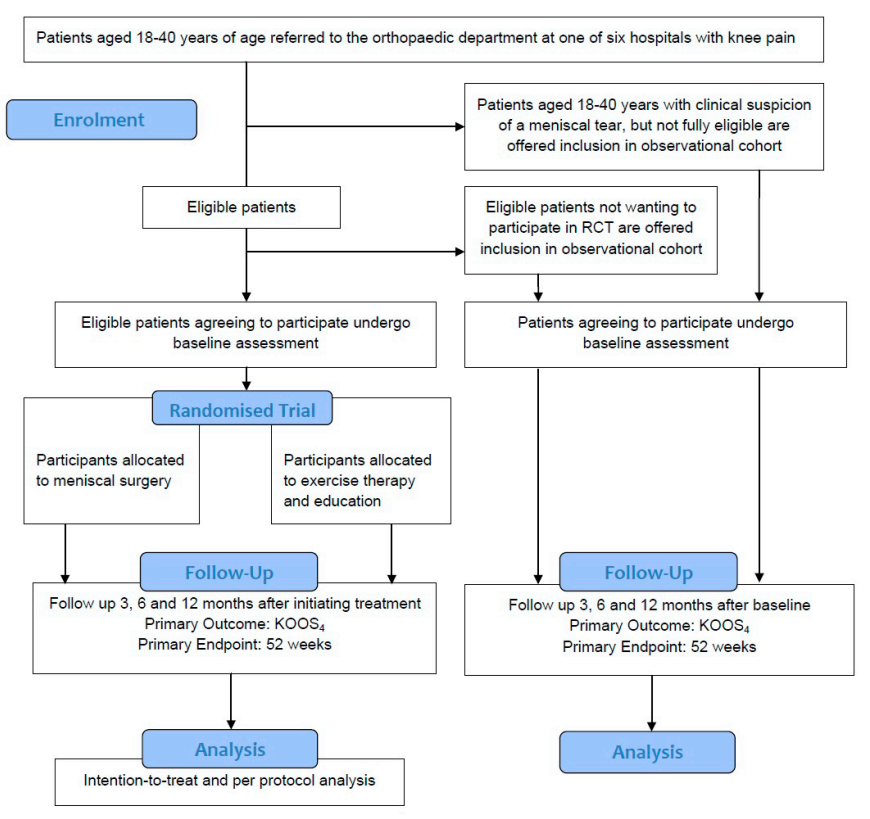

Figure 1 Patient flow through the study. RCT, randomised controlled trial; $\mathrm{KOOS}_{4}$, the mean score for the Knee Injury and Osteoarthritis Outcome Score subscales pain, symptoms, function in sports and recreational activities (Sport/Rec) and quality of life (QOL).

surgery (ie, repair or resection), but exercise might be a valid treatment option considering previous trials in middle-aged and older patients. ${ }^{57}$ Evidence from more severe traumatic knee injuries such as anterior cruciate ligament (ACL) tears suggest that more than half of ACL-injured patients can obtain satisfactory knee function with structured and supervised exercise therapy as first-line treatment before considering surgery. ${ }^{10}$

The aim of this RCT is to investigate if early arthroscopic meniscal surgery is superior to individualised supervised exercise therapy and patient education, with the option of later surgery if needed, in improving pain, function and quality of life in young patients (18-40 years of age) with meniscal tears. We hypothesise that patients randomised to surgery will improve significantly more in pain, function and quality of life after 12 months than those randomised to exercise and patient education.

\section{METHODS AND ANALYSIS \\ Study design}

This study protocol (version 2, 30 March 2017) describe the design of a multicentre, parallel-group RCT (1:1 ratio) conducted at six orthopaedic departments across all five healthcare regions in Denmark.

The study protocol conforms with the Standard Protocol Items: Recommendations for Interventional Trials (SPIRIT) ${ }^{11}$ while the RCT will conform to the Consolidated Standards of Reporting Trials (CONSORT) statement for reporting RCTs. ${ }^{12}$

The orthopaedic departments at Aalborg University Hospital, Aarhus University Hospital, Lillebælt Hospital
(Kolding), Næstved Hospital and Copenhagen University Hospital (Amager-Hvidovre) will recruit patients for the study.

The study is registered at ClinicalTrials.gov in December 2016 (NCT02995551) and enrolment started at the first hospital in January 2017 and at the last hospital in April 2017 and recruitment is expected to finalise in June 2018.

\section{Patients}

One hundred and forty patients fulfilling the eligibility criteria below will be included.

\section{Inclusion criteria}

- Adults aged 18-40 years with knee pain.

- Clinical history and symptoms consistent with meniscal tear and meniscal tear verified on MRI.

- Deemed eligible for meniscal surgery (ie, repair or resection) by the examining orthopaedic surgeon.

- Willing to participate in 12 weeks of supervised exercise twice a week and undergo surgery for the meniscal tear as soon as possible.

\section{Exclusion criteria}

- Previous knee surgery on the affected knee.

- Clinical suspicion (acute locking of knee and/or extension deficit) of displaced bucket-handle tear confirmed by MRI.

- Fracture of the affected extremity within the previous 6 months.

- Complete rupture of one or more knee ligaments.

- Participation in supervised systematic exercise for knee problems within the last 3 months prior to recruitment.

- Other reasons for exclusion (unable to understand Danish, mentally unable to participate, etc).

\section{Recruitment procedure}

The overall trial flow is outlined in figure 1. Patients referred to the orthopaedic department at one of the six hospitals and complying with the eligibility criteria will be invited to participate in the RCT study. Orthopaedic surgeons, nurses and physiotherapists involved in recruitment have been trained and instructed in the recruitment procedure in order to maximise the recruitment rate.

Oral and written information about the study will be provided by the examining orthopaedic surgeon and/or by a nurse or physiotherapist at the six different recruiting orthopaedic departments in an undisturbed room during consultation at the hospital. The study information is also available in a video for the patient to watch at home or at the hospital, before deciding to participate or not. Patients are recommended to take at least 24 hours to consider and discuss participation with a relative or a lay representative before deciding on participation in the study.

Either the local project worker or a central study coordinator (depending on local differences at the hospitals) will contact the patient and ask whether they are willing 
to participate in the study. If so, written informed consent will be obtained (see online supplementary file 1).

\section{Randomisation procedure and concealment of allocation}

Patients fulfilling eligibility criteria and willing to participate will be randomised after baseline assessment (1:1 allocation ratio). A priori, an independent statistician has prepared a computer-generated randomisation schedule in random-sized permuted blocks of four or six patients stratified by hospital and gender to ensure that the number of participants receiving the two interventions is closely balanced within each stratum. The allocation numbers will be concealed in opaque sealed envelopes prepared by a central study coordinator. The envelopes will only be accessible by the central study coordinator, only opening them after informed consent and baseline measures have been obtained.

\section{Blinding}

An independent statistician blinded to group allocation will perform the primary RCT analysis. To reduce risk of interpretation bias, blinded results from the analyses (Group A compared with Group B) will be presented to all authors, who will agree on two alternative written interpretations before the data manager unblinds the randomisation code. ${ }^{13}$

\section{Observational cohort}

Patients fulfilling all eligibility criteria, but unwilling to participate in the randomised study, and patients aged 18-40 years with a clinical history and symptoms consistent with a meniscal tear, but not fulfilling the other eligibility criteria, are invited into an observational cohort with the same self-reported questionnaires as applied in the RCT, but following usual clinical practice. Written informed consent will be obtained for all cohort participants.

\section{Interventions}

Patients will be randomised to one of two treatments initiated as soon as possible after randomisation.

\section{Arthroscopic meniscal surgery}

Arthroscopic meniscal repair or resection will be conducted at the discretion of the operating surgeon at one of the six hospitals. The specific surgical procedure (ie, repair or resection) cannot be determined before the surgeon has visual confirmation about the exact knee pathology and extent of the meniscal tear at arthroscopy. Patients will receive the standard postoperative rehabilitation depending on type of surgery (ie, repair or resection). A standard leaflet with exercises will be given to patients undergoing APM at all surgery sites. Patients undergoing meniscal repair will follow a hospital-specific rehabilitation regimen to improve the external validity of the study findings. The hospital-specific regimes range from postsurgical control of range of motion and instruction in a standardised postsurgical exercise programme to referral to supervised, knee-related exercises focusing on increasing range of motion and strength, most often in patients with reduced range of motion or not able to activate vastus medialis following meniscal repair.

\section{Exercise therapy and patient education}

Patients allocated to exercise therapy and patient education will twice weekly participate in a 12-week individualised, supervised exercise programme (approximately $60-90 \mathrm{~min} /$ session) tailored to $18-40$ years old patients with meniscal tear. The content of the exercise therapy programme was guided by available evidence from patients with other types of knee injuries and osteoarthritis $^{71014-17}$ and developed in close collaboration with experienced physical therapists and pilot patients. ${ }^{18}$ Each exercise session includes a warm up period of $5 \mathrm{~min}$ on a stationary bike and neuromuscular and strengthening exercises focusing on the lower extremities. If needed during the first weeks, two exercises focusing on reducing swelling and increasing range of motion is included. The neuromuscular exercises are individualised based on 2-6 levels of difficulty and performed with 10-15 repetitions in 2-3 sets. The strengthening exercises are initially performed in two sets of 15 repetitions, progressing to three sets of 12, three sets of 10 and three sets of eight repetitions. ${ }^{18}$ The exercise therapy programme was tested in a pilot study ${ }^{18}$ and reported according to the Consensus on Exercise Reporting Template (CERT). ${ }^{19}$ The pilot study included six patients fulfilling the same eligibility criteria as for the present RCT. The exercise programme was found feasible with few short-lasting periods of increased pain or other symptoms during the exercises and marked self-reported improvements after 12 weeks. ${ }^{18}$ For full details about the exercise programme please refer to Skou and Thorlund. ${ }^{18}$

The patient education was developed to support the exercise therapy programme and build motivation and capability to sustain the exercise after the 12-week programme. It was adapted, through interviews with pilot study participants, based on our experiences from the Good Life with osteoArthritis in Denmark (GLA:D) programme for patients with knee and hip pain. ${ }^{17}$ See further description in table 1 according to the Template for Intervention Description and Replication (TIDieR). ${ }^{20}$

The exercise therapy programme and patient education will be delivered at one of 16 private physiotherapy clinics and municipalities geographically related to the six hospitals. All physiotherapists are part of the GLA:D infrastructure and used to deliver and supervise exercise therapy in a similar manner as in the present study. Furthermore, all physiotherapists attended a specific half-day course to be certified to deliver the specific treatment in this trial.

\section{Crossover and discontinuation}

Crossovers are common in studies randomising patients to surgical or non-surgical treatment. ${ }^{14}{ }^{14}$ Based on previous experience, a number of precautions have been taken to reduce crossover and discontinuation. Within 
Table 1 Overview of the patient education*

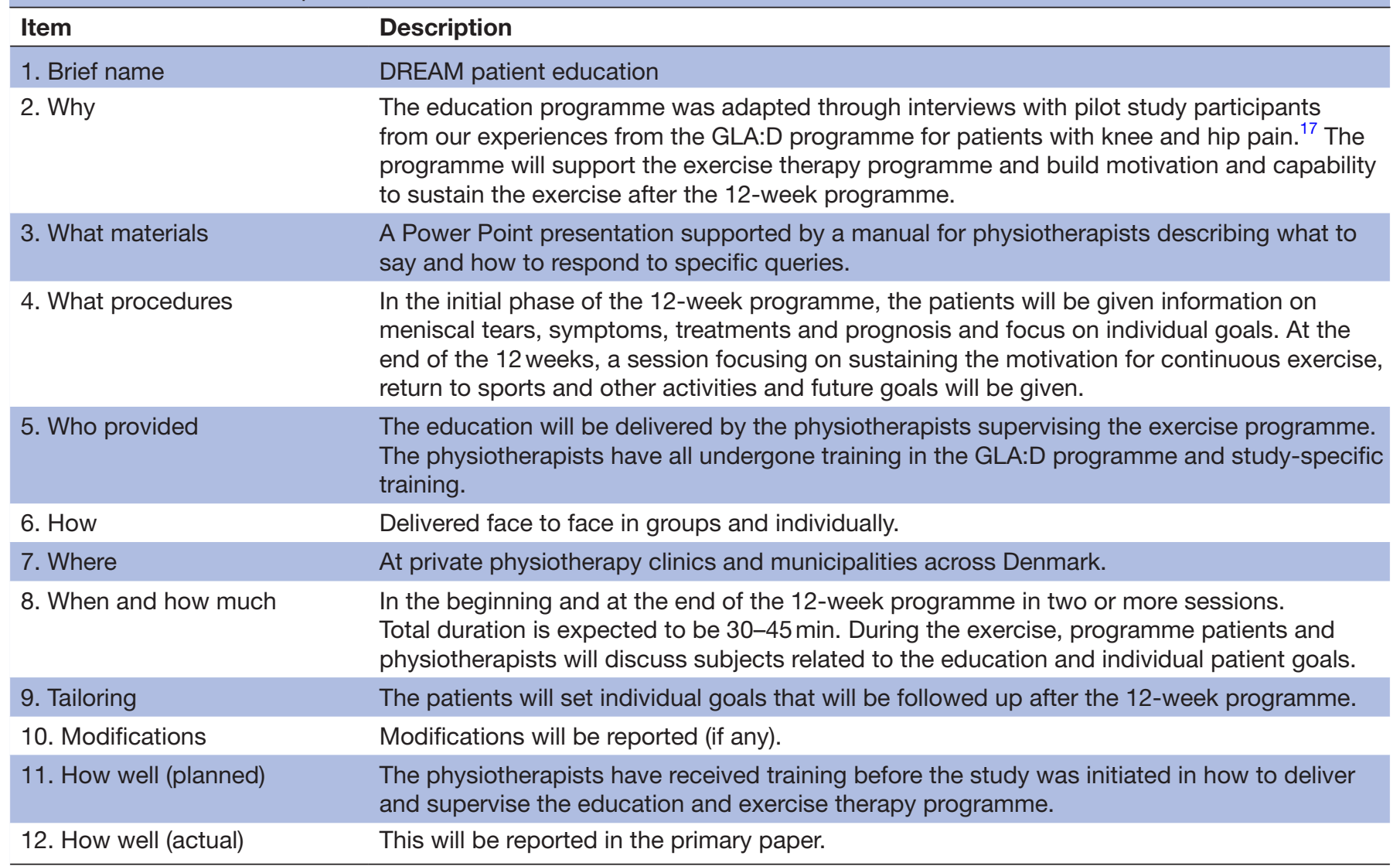

*Described according to the Template for Intervention Description and Replication (TIDieR). ${ }^{20}$

DREAM, Danish Rct on Exercise versus Arthroscopic Meniscal surgery for young adults; GLA:D, Good Life with osteoArthritis in Denmark.

1 week from randomisation, the central study coordinator will call patients and talk to them about their participation in the study and what is going to happen during the study. The physiotherapists have been trained to encourage patients to stay in the exercise and education group at least until after the 12-week programme has been completed. This is important as it often require around 6 weeks before important improvements in pain are reported. ${ }^{22}$ Results from the pilot study suggest that clinically important improvements might not occur until after 8-10 weeks. ${ }^{18}$ A similar time frame of improvements can be expected for patients undergoing surgery. Patients insisting to crossover to surgery or discontinue their participation will be contacted by the central study coordinator regarding their reasons for crossover or discontinuation. If needed, they will be reassessed by an orthopaedic surgeon. The reason for each crossover and discontinuations will be registered. Patients crossing over will remain in the study and will be included in the intention-to-treat analyses.

The general crossover criteria from exercise and education to surgery are:

- score of 25 or less on the pain and/or quality of life (QOL) subscale on the Knee Injury and Osteoarthritis Outcome Score (KOOS) ${ }^{23} 24$ or, agreement between patient and orthopaedic surgeon that surgery is necessary.

\section{Data collection procedure}

Data will be collected at baseline, at surgery (for those randomised to surgery) and 3,6 and 12 months after initiating the treatment. All but the physical performance tests will be collected using online-based questionnaires or Short Message Services (SMS). Physical performance measures will be assessed at the hospitals by project workers specifically trained in the test protocol. Please refer to table 2 for an overview of collection of the different outcomes.

\section{Outcomes}

Baseline characteristics

Patient characteristics such as height, weight, pain location, symptom duration and symptom onset will be collected. Symptom duration and symptom onset will be answered by the questions: 'How long have you had your knee pain/problems for which you are now having treatment?' with six response options ranging from 'less than 2 weeks' to 'more than 24 months', 'How did the knee pain/problems for which you are now having treatment develop?' with three response options 'The pain/ 
Table 2 Overview of data collection*

\begin{tabular}{|c|c|c|c|c|c|}
\hline & Baseline & Surgery & 3 month & 6 month & 12 month \\
\hline \multicolumn{6}{|l|}{ Baseline characteristics } \\
\hline Age & $x$ & & & & \\
\hline Gender & $x$ & & & & \\
\hline Study knee & $x$ & & & & \\
\hline Height & $x$ & & & & \\
\hline Weight & $x$ & & $x$ & $x$ & $\mathrm{x}$ \\
\hline Education level & $x$ & & & & \\
\hline Employment status & $x$ & & & & \\
\hline Prior treatment of knee & $x$ & & & & \\
\hline Smoking status & $x$ & & & & \\
\hline Comorbidities & $x$ & & & & \\
\hline Symptom duration & $x$ & & & & \\
\hline Symptom onset & $x$ & & & & \\
\hline $\begin{array}{l}\text { Joint line tenderness (medial } \\
\text { and lateral) }\end{array}$ & $x$ & & & & \\
\hline $\begin{array}{l}\text { Thessaly's test (at } 20^{\circ} \text { knee } \\
\text { flexion) }\end{array}$ & $x$ & & & & \\
\hline McMurray's test & $x$ & & & & \\
\hline
\end{tabular}

\section{Surgery information}

ISAKOS meniscal tear

classification

\begin{tabular}{|c|c|c|c|c|}
\hline Surgery reports & $x$ & & & \\
\hline Patient reported outcomes & & & & \\
\hline KOOS & $x$ & $x$ & $x$ & $x$ \\
\hline WOMET & $x$ & $x$ & $x$ & $\mathrm{x}$ \\
\hline EQ-5D & $x$ & $x$ & $x$ & $x$ \\
\hline Physical activity level & $x$ & $x$ & $x$ & $x$ \\
\hline Sports participation & $x$ & $x$ & $x$ & $x$ \\
\hline Pain location & $x$ & $x$ & $x$ & $\mathrm{x}$ \\
\hline $\begin{array}{l}\text { Symptoms of catching and } \\
\text { locking }\end{array}$ & $x$ & $x$ & $x$ & $x$ \\
\hline Knee instability & $x$ & $x$ & $x$ & $\mathrm{x}$ \\
\hline Global perceived effect & & $x$ & $x$ & $x$ \\
\hline $\begin{array}{l}\text { Patient acceptable symptom } \\
\text { state }\end{array}$ & & $x$ & $x$ & $x$ \\
\hline Treatment failure & & & & $x$ \\
\hline Physical performance tests & & & & \\
\hline Isometric muscle strength & $x$ & $x$ & & $x$ \\
\hline Knee-bend test & $x$ & $x$ & & $x$ \\
\hline Jump performance (two tests) & & & & $x$ \\
\hline Adverse events & & & & \\
\hline Patient-reported at follow-up & & $x$ & $x$ & $x$ \\
\hline Medical record review & & & & $x$ \\
\hline Treatment-related variables & & & & \\
\hline
\end{tabular}

Continued 
Table 2 Continued

\begin{tabular}{llll}
\hline \multicolumn{1}{c}{ Baseline } & Surgery & 3month & 6month \\
\hline $\begin{array}{l}\text { Compliance with and } \\
\text { progression of exercise (only for } \\
\text { patients in the exercise group, } \\
\text { registered after each session) }\end{array}$ & & \\
$\begin{array}{l}\text { Participation in postsurgery } \\
\text { exercise (only for patients in the } \\
\text { surgery group) }\end{array}$ & & \\
$\begin{array}{l}\text { Surgery and/or other treatments } \\
\text { during follow-up }\end{array}$ & & \\
\hline
\end{tabular}

*Some of the other outcome measures will be collected using Short Message Services and are not shown as they follow a different follow-up pattern.

Data in the surgery column are only collected for patients undergoing surgery.

EQ-5D, EuroQol Group 5-Dimension Self-Report Questionnaire; ISAKOS, International Society of Arthroscopy, Knee Surgery and Orthopaedic Sports Medicine, classification of meniscal tears questionnaire; KOOS, Knee injury and Osteoarthritis Outcome Score; WOMET, Western Ontario Meniscal Evaluation Tool.

problems have slowly developed over time', 'As a result of a less severe incident (ie, kneeling, sliding, and/or twisting of the knee or the like)', and 'As a result of a severe incident (ie, during sports, a crash, or a collision or the like)'.

\section{Primary outcome}

The primary outcome will be the between-group difference in change in $\mathrm{KOOS}_{4}$ between the group randomised to meniscal surgery and the group randomised to exercise therapy and patient education from baseline to 12 months follow-up. $\mathrm{KOOS}_{4}$ is the mean score for the KOOS subscales pain, symptoms, function in sports and recreational activities (Sport/Rec) and quality of life (QOL); the same score was used in a trial comparing surgery to supervised exercise as treatment for ACL tears in patients of similar age as in the present trial. ${ }^{10} \mathrm{KOOS}$ subscale scores range from 0 (worst) to 100 (best). ${ }^{23} 24$ KOOS is a validated knee-specific questionnaire used to assess patient-reported outcomes in the continuum from knee injury (including meniscal tears) to osteoarthritis and is widely used across the world. ${ }^{23-25}$

\section{Secondary outcomes}

\section{KOOS subscales}

To allow for clinical in-depth interpretation, the primary outcome will be complemented by the five individual KOOS subscales (ie, including the fifth subscale-activities of daily living (ADL) subscale). ${ }^{23} 24$

\section{Western Ontario Meniscal Evaluation Tool (WOMET)}

WOMET $^{26} 27$ is a meniscus specific valid, reliable and responsive patient-reported outcome measure that will be used to complement the KOOS score.

\section{Physical performance}

Isometric muscle strength will be assessed using the reliable and valid FysioMeter ${ }^{28}$ functional performance measured using the maximum number of knee-bends in $30 \mathrm{~s}$, the one-leg hop for distance and the $6 \mathrm{~m}$ timed hop previously applied in trials comparing meniscal surgery to exercise. ${ }^{7}$ The two hop tests will only be assessed at 12 months due to risk of re-injury.

\section{Adverse events}

Adverse events (AE) and serious adverse events (SAE) will be recorded at all follow-ups by asking patients about potential AEs using open-probe questioning to ensure that all AEs are recorded. Furthermore, the medical records from the participating hospitals will be checked at the primary endpoint (12 months) for all AEs occurring from inclusion until the 12 months follow-up. An $\mathrm{AE}$ is defined as any undesirable experience during follow-up leading to contact with the healthcare system (general practitioner or hospital). If an $\mathrm{AE}$ result in hospitalisation, prolonged inpatient hospital care, result in re-surgery, or if an $\mathrm{AE}$ is life-threatening, result in death, permanent disability or damage, they will be categorised as SAEs. ${ }^{29} \mathrm{SAE}$ will include cardiovascular or gastrointestinal events, pulmonary embolism, systemic or local infection (or treatment with antibiotics) and deep vein thrombosis, but also other AEs adhering to the definition above will be categorised as an SAE. Crossover to surgery will not be considered as an adverse event as the study is comparing two treatment strategies: early surgery or early exercise and education with the possibility of later surgery. However, crossover to surgery will be registered and reported as it is important when evaluating the clinical applicability of the results. AEs will be categorised into index knee or other sites and will be recorded and assessed for severity by the adjudication committee independent of whether or not there is a causal relationship with study treatments. For all AEs, date of healthcare system contact will be registered. Furthermore, duration of SAEs and potential consequences of SAEs will be assessed. 
Other outcomes measures

\section{$E Q-5 D-5 L$}

General health will be assessed using the reliable and valid EQ-5D-5L questionnaire (5-level version), both the descriptive index and the EQ-VAS. ${ }^{30} 31$ This will also allow for a later cost-effectiveness analysis.

\section{Physical activity and sports}

Information on physical activity level ${ }^{32}$ and participation in sports (Tegner activity scale) ${ }^{33}$ will be collected.

\section{Pain location}

Patients are asked about pain in other body parts than the knee during the last week with the categories: foot or lower leg, thigh and hip, back, neck or shoulders and arm or hand, all answered by 'yes' or 'no'.

\section{Symptoms of catching and locking}

Patients will report the presence and frequency of mechanical knee symptoms (ie, the sensation of catching and locking) based on the question: 'How often have you experienced catching or locking of the knee that is about to undergo treatment?' with five response options ranging from 'never' to 'daily'.

\section{Knee instability}

Patients will report the presence and frequency of knee instability based on the question: 'In the last month, have you felt that your knee was unstable or was about to buckle' with six response options ranging from 'never' to 'all the time'. Subsequently, the patients are asked to what extent the knee instability have affected their daily activity level in the last month answered by one of six response options ranging from 'my knee is not unstable or about to buckle' to 'the symptoms keeps me away from all activities of daily living'.

\section{Global perceived effect, patient acceptable symptom state and treatment failure}

Global perceived effect (GPE) will be assessed with the question: How are your knee problems now compared with before you entered this study? Answered on a sevenpoint Likert scale ranging from 'Improved, an important improvement' to 'Worse, an important worsening'. Satisfaction with current knee function (ie, patient acceptable symptom state (PASS)) will be assessed with the question: "When you think of your knee function, will you consider your current condition as satisfying? By knee function, you should take into account your activities of daily living, sport and recreational activities, your pain and other symptoms and your quality of life'. Answered by 'yes' or 'no'. This question has previously been used to assess PASS $^{34}$ in patients with knee injury. ${ }^{35}$ Participants not satisfied with current knee function (ie, answering 'no' to the PASS question) will be asked to complete a second single-item question, relating to treatment failure (TF) at the 12-month follow-up: 'Would you consider your current state as being so unsatisfactory that you think the treatment has failed?'. Answered by 'yes' or 'no'.35
Pain and function during follow-up

Using SMS, all patients in the RCT will receive a questionnaire each week during the first 3 months and after that each month until the 12 months follow-up with questions from the subscale Sport/Rec from the KOOS 2324 and a question regarding pain intensity when ascending/ descending stairs and a question regarding pain intensity during sitting/lying on a 11-point Numeric Rating Scale (NRS). ${ }^{36}$ Patients in the observational cohort will only receive the SMS monthly until the 12 months follow-up. Patients in the group randomised to exercise and education will, in addition, register pain intensity on a 11-point $\mathrm{NRS}^{36}$ before and after each of the supervised exercise sessions.

\section{Treatment-related outcomes}

\section{Information on knee pathology and type of surgery}

Information about location of tear, type of tear and type of treatment (repair or resection) will be collected using an expanded version of the International Society of Arthroscopy, Knee Surgery and Orthopaedic Sports Medicine (ISAKOS) classification of meniscal tears questionnaire by the operating surgeon, ${ }^{37}$ including International Cartilage Repair Society (ICRS) grading of cartilage damage. ${ }^{38}$ This will be supplemented with data from surgery reports on specific pathological findings and surgical procedures carried out.

\section{Compliance, exercise level/intensity and other treatments received} during the study

Compliance with the supervised exercise sessions (ie, number of sessions out of 24 possible sessions) and progression of the exercises will be registered for patients in the group randomised to exercise and education. Poor compliance is defined as participating in less than 18 of the 24 exercise sessions. Any participation in supervised exercise postsurgery will be self-reported using an online questionnaire in the group randomised to surgery. Surgery of the knee during follow-up will be registered through a review of the medical records.

Furthermore, patients will be asked to report other treatments related to the knee that they received during follow-up.

\section{Data management}

The data collection and management procedures have been approved by the Danish Data Protection Agency (University of Southern Denmark, 16/45314). Personal information about patients will be kept separate from the main dataset and will not be shared. All personal data will be stored securely in order to protect confidentiality before, during and after the trial. Data entry and coding of the de-identified data will be conducted by trained staff at the University of Southern Denmark.

\section{Data monitoring}

The study will not have a formal data monitoring committee as adverse events of treatments are well known due to their wide application in the healthcare sector. 
Any unexpected serious adverse events or outcomes will be discussed by the trial management committee (identical to the authors of this protocol). Furthermore, the trial management committee will monitor recruitment, treatment and attrition rates and any concerns related to the study.

\section{Adjudication committee}

An adjudication committee, comprising members with prior adjudication experience, will independently adjudicate all adverse events in the RCT as to whether they are serious adverse events or not and categorise them into subcategories. They will each receive the adverse events in raw format after the last patient has undergone the 12 month follow-up. Any disagreements between the adjudication committee members will be resolved by consensus. If consensus cannot be reached, additional information will be requested from the hospitals.

\section{Sample size calculation}

The study will be powered to detect a difference in change of 10 points between the surgical and non-surgical group in the primary outcome $\left(\mathrm{KOOS}_{4}\right)$ from baseline to 12 months follow-up. A 10-point difference in change between groups in $\mathrm{KOOS}_{4}$ is considered clinically relevant and a similar cut-off has previously been used in trials comparing surgery for different knee pathologies to non-operative treatment. ${ }^{101439}$ To detect this difference, 59 patients in each of the intervention groups is needed (assuming a common SD of 16.5 , power $=90 \%$, alpha level $=0.05)$. We plan to recruit a total of 140 patients to account for loss to follow-up (19\%). Based on Danish National Patient Registry data from 2013 to 2014, more than a total of 800 primary meniscal procedures are carried out in patients aged 18-40 years at the participating recruitment sites. Patients for the observational cohort will be included consecutively until inclusion in the RCT has been completed or until 1000 patients has been included.

\section{Stopping rule}

If the intended sample size is not reached at 30 months after recruitment has started at all participating hospitals, the inclusion of patients will stop at 106 patients, which will ensure a power of $80 \%$ anticipating $20 \%$ loss to follow-up.

\section{Statistical analysis}

Between-group comparisons of change from baseline to 1 year follow-up in the primary and secondary continuous outcomes (ie, KOOS, WOMET, muscle strength, kneebend test and jump performance) will be analysed using a repeated measures mixed model with patients as random effect, visit (baseline, 3, 6 and 12 months) and treatment arm (meniscal surgery or exercise and education) as fixed effects, and with adjustment for baseline imbalance and randomisation stratification factors, that is, hospital and gender. No imputation will take place. A CI excluding 10 points or more in the $\mathrm{KOOS}_{4}$ score will be interpreted as a lack of a clinical meaningful difference. Differences in the trajectories of $\mathrm{KOOS}_{4}$ scores from baseline to 12 months will be analysed using a random slopes and intercepts model. The occurrence of adverse events will be compared between groups at the 1 year follow-up using a Poisson regression model with a robust error variance. ${ }^{40}$ Categorical secondary outcomes will be analysed using $\mathrm{X}^{2}$ test, Fisher's exact test or Mann-Whitney U test as appropriate.

All randomised patients will be included in the intention to treat analysis and in the safety analysis. Per protocol and as treated analyses will be performed for the primary outcome. Those who crossover to surgery or have poor compliance with the exercise in the exercise and education group and those who do not undergo surgery in the surgery group are excluded from the per protocol analysis, while the as-treated analysis is expected to have three groups, that is, including the group crossing over to surgery in addition to the original two randomisation groups.

A detailed statistical analysis plan will be made publicly available before unblinding the data and any analyses are performed.

\section{ETHICS, DISSEMINATION AND PERSPECTIVES OF THE STUDY Ethics and auditing}

The study is approved by the Regional Committees on Health Research Ethics for Southern Denmark (S-20160151) and will be conducted in agreement with the Helsinki declaration. Informed consent material is available in Danish with the approved protocol. If a patient sustains any trial-related harm they are covered by Danish law. The Regional Committees on Health Research Ethics are annually selecting a number of studies for auditing. The audit process is independent of investigators and sponsors.

\section{Dissemination and protocol amendments}

The primary RCT results will be submitted for publication to an international, peer-reviewed journal, regardless of whether the results are positive, negative or inconclusive in relation to the study hypothesis. Authorship eligibility will be based on the recommendations from the International Committee of Medical Journal Editors (ICMJE).

Any important protocol amendments will be reported to the Regional Committees on Health Research Ethics for Southern Denmark, registered at ClinicalTrials.gov and communicated in the primary RCT report.

\section{Perspectives of the study}

The results of this RCT will either provide high-quality evidence supporting a practice of early meniscal surgery for young adults aged 40 years or younger with meniscal tears or support a practice of initial supervised exercise therapy and patient education with the option of later surgery if needed in some patients. Either way, the results will provide scientific support for doctors and patients 
when discussing the optimal treatment option for the individual patient.

\section{Author affiliations}

${ }^{1}$ Department of Sports Science and Clinical Biomechanics, Research Unit for Musculoskeletal Function and Physiotherapy, University of Southern Denmark, Odense, Denmark

${ }^{2}$ Department of Physiotherapy and Occupational Therapy, Næstved-SlagelseRingsted Hospitals, Slagelse, Denmark

${ }^{3}$ Department of Sports Traumatology, Aarhus University Hospital, Aarhus, Denmark ${ }^{4}$ Department of Orthopaedic Surgery, Sports Orthopedic Research CenterCopenhagen (SORC-C), Copenhagen University Hospital, Copenhagen, Denmark ${ }^{5}$ Department of Orthopaedic Surgery, Aalborg University Hospital, Aalborg, Denmark ${ }^{6}$ Department of Orthopaedic Surgery, Lillebælt Hospital in Kolding, Kolding, Denmark ${ }^{7}$ Department of Regional Health Research, University of Southern Denmark, Odense, Denmark

${ }^{8}$ Department of Orthopaedic Surgery, Næstved Hospital, Næstved, Denmark ${ }^{9}$ Department of Orthopaedics and Traumatology, Odense University Hospital, Odense, Denmark

Twitter Follow Søren T. Skou at @STSkou and Jonas B. Thorlund at @jbthorlund.

Acknowledgements We thank the Orthopaedic surgeons and other healthcare personnel involved in the recruitment, test and treatment of patients at the Department of Orthopaedic Surgery, Copenhagen University Hospital, AmagerHvidovre; the Department of Orthopaedic Surgery, Lillebælt Hospital in Kolding; the Department of Orthopaedic Surgery, Næstved Hospital; the Department of Orthopaedics and Traumatology, Odense University Hospital; the Department of Orthopaedic Surgery, Aalborg University Hospital and the Department of Sports Traumatology, Aarhus University Hospital and physiotherapists treating patients in the exercise and education group from Arkadens Fysioterapi, Benefit Hobro, Brønshøj Fysioterapi \& Trænings center, Charlottehøj Fysioterapi, FabrikkenSlagelse, Fysikken Ringsted, Fysio Danmark Holbæk, Fysio Danmark Odense, Fysioterapi \& Træningsklinik Frederiksberg, Fysioterapi \& Motion Hvidovre, Fysiovejen, Fysium Næstved, Faaborg Fysioterapi, Guldborgsund Kommune, Kolding Fysioterapi, Middelfart Fysioterapi and Aars Fysioterapi. Statistical consultant for study planning and final data analysis is Professor Jonas Ranstam. Central study coordinator is Anne Marie Rosager. Study advisory board to consult on study planning, results interpretation and other relevant matters: Ewa Roos, University of Southern Denmark; Stefan Lohmander, Lund University; Teppo Järvinen, University of Helsinki; Martin Englund, Lund University; Andrew Carr, University of Oxford.

Contributors STS and JBT conceived the trial and led the development of all procedures including intervention design (exercise intervention and patient education), data management and statistical analyses and drafted the first version of the manuscript. ML, PH, HPJ, CJ, MA and UJ provided feedback on the study, led setup of procedures and data collection at the recruiting hospitals. All authors provided critical intellectual input to the manuscript and read and approved the final version of the manuscript, agreeing to be accountable for all aspects of the work.

Funding The study is funded by the Danish Council for Independent Research (DFF-6110-00045), IMK Almene Fond, The Lundbeck Foundation, the Spar Nord Foundation, the Danish Rheumatism Association, The Association of Danish Physiotherapists Research Fund and The Research council at Næstved-SlagelseRingsted Hospitals. The funders have no role in the design of the study and will not have any role in its execution, data management, analysis and interpretation or on the decision to submit results for publication.

Competing interests STS is one of the founders of Good Life with osteoarthritis in Denmark (GLA:D), which is a non-profit initiative hosted at University of Southern Denmark. STS is Associate Editor for Journal of Orthopaedic and Sports Physical Therapy. The authors affirm that they have no other competing interests.

Patient consent Detail has been removed from this case description/these case descriptions to ensure anonymity. The editors and reviewers have seen the detailed information available and are satisfied that the information backs up the case the authors are making.

Ethics approval The Regional Committees on Health Research Ethics for Southern Denmark.

Provenance and peer review Not commissioned; externally peer reviewed.

Open Access This is an Open Access article distributed in accordance with the Creative Commons Attribution Non Commercial (CC BY-NC 4.0) license, which permits others to distribute, remix, adapt, build upon this work non-commercially, and license their derivative works on different terms, provided the original work is properly cited and the use is non-commercial. See: http://creativecommons.org/ licenses/by-nc/4.0/

(C) Article author(s) (or their employer(s) unless otherwise stated in the text of the article) 2017. All rights reserved. No commercial use is permitted unless otherwise expressly granted.

\section{REFERENCES}

1. Lim HC, Adie S, Naylor JM, et al. Randomised trial support for orthopaedic surgical procedures. PLoS One 2014;9:e96745.

2. van Adrichem RA, Nemeth B, Algra A, et al. Thromboprophylaxis after knee arthroscopy and lower-leg casting. N Engl J Med 2017;376:515-25.

3. Kim S, Bosque J, Meehan JP, et al. Increase in outpatient knee arthroscopy in the United States: a comparison of National surveys of ambulatory surgery, 1996 and 2006. J Bone Joint Surg Am 2011;93:994-1000.

4. Price A, Beard D. Arthroscopy for degenerate meniscal tears of the knee. BMJ 2014;348:g2382.

5. Thorlund JB, Juhl CB, Roos EM, et al. Arthroscopic surgery for degenerative knee: systematic review and meta-analysis of benefits and harms. BMJ 2015;350:h2747.

6. Khan M, Evaniew N, Bedi A, et al. Arthroscopic surgery for degenerative tears of the meniscus: a systematic review and metaanalysis. CMAJ 2014;186:1057-64.

7. Kise NJ, Risberg MA, Stensrud S, et al. Exercise therapy versus arthroscopic partial meniscectomy for degenerative meniscal tear in middle aged patients: randomised controlled trial with two year follow-up. BMJ 2016;354:i3740.

8. Thorlund JB, Østengaard L, Cardy N, et al. Trajectory of selfreported pain and function and knee extensor muscle strength in young patients undergoing arthroscopic surgery for meniscal tears: a systematic review and meta-analysis. J Sci Med Sport 2017;20:712-7.

9. Buchbinder R, Harris IA, Sprowson A. Management of degenerative meniscal tears and the role of surgery. BMJ 2015;350:h2212.

10. Frobell RB, Roos EM, Roos HP, et al. A randomized trial of treatment for acute anterior cruciate ligament tears. N Engl J Med 2010;363:331-42.

11. Chan AW, Tetzlaff JM, Gøtzsche PC, et al. SPIRIT 2013 explanation and elaboration: guidance for protocols of clinical trials. BMJ 2013;346:e7586.

12. Moher D, Hopewell S, Schulz KF, et al. CONSORT 2010 explanation and elaboration: updated guidelines for reporting parallel group randomised trials. BMJ 2010;340:c869.

13. Järvinen TL, Sihvonen R, Bhandari M, et al. Blinded interpretation of study results can feasibly and effectively diminish interpretation bias. J Clin Epidemiol 2014;67:769-72.

14. Skou ST, Roos EM, Laursen MB, et al. A randomized, controlled trial of total knee replacement. N Engl J Med 2015;373:1597-606.

15. Ageberg E, Roos EM. Neuromuscular exercise as treatment of degenerative knee disease. Exerc Sport Sci Rev 2015;43:14-22.

16. Skou ST, Rasmussen S, Laursen MB, et al. The efficacy of 12 weeks non-surgical treatment for patients not eligible for total knee replacement: a randomized controlled trial with 1-year follow-up. Osteoarthritis Cartilage 2015;23:1465-75.

17. Skou ST, Roos EM. Good life with osteoArthritis in Denmark (GLA:DTM): evidence-based education and supervised neuromuscular exercise delivered by certified physiotherapists nationwide. BMC Musculoskelet Disord 2017;18:72.

18. Skou ST, Thorlund JB. A 12-week supervised exercise therapy program for young adults with a meniscal tear: Program development and feasibility study. J Bodyw Mov Ther 2017.

19. Slade SC, Dionne CE, Underwood M, et al. Consensus on Exercise Reporting Template (CERT): explanation and elaboration statement. Br J Sports Med 2016:50:1428-37.

20. Hoffmann TC, Glasziou PP, Boutron I, et al. Better reporting of interventions: Template for Intervention Description and Replication (TIDieR) checklist and guide. BMJ 2014;348:g1687.

21. Weinstein JN, Tosteson TD, Lurie JD, et al. Surgical vs nonoperative treatment for lumbar disk herniation: the Spine Patient Outcomes Research Trial (SPORT): a randomized trial. JAMA 2006;296:2441-50.

22. Sandal LF, Roos EM, Bøgesvang SJ, et al. Pain trajectory and exercise-induced pain flares during 8 weeks of neuromuscular 
exercise in individuals with knee and hip pain. Osteoarthritis Cartilage 2016;24:589-92.

23. Collins NJ, Prinsen CA, Christensen R, et al. Knee Injury and Osteoarthritis Outcome score (KOOS): systematic review and meta-analysis of measurement properties. Osteoarthritis Cartilage 2016;24:1317-29.

24. Roos EM, Roos HP, Lohmander LS, et al. Knee Injury and Osteoarthritis Outcome score (KOOS) - development of a selfadministered outcome measure. J Orthop Sports Phys Ther 1998;28:88-96.

25. Thorlund JB, Englund M, Christensen R, et al. Patient reported outcomes in patients undergoing arthroscopic partial meniscectomy for traumatic or degenerative meniscal tears: comparative prospective cohort study. BMJ 2017;356:j356.

26. Kirkley A, Griffin S, Whelan D. The development and validation of a quality of life-measurement tool for patients with meniscal pathology: the Western Ontario Meniscal Evaluation Tool (WOMET). Clin J Sport Med 2007;17:349-56.

27. Sihvonen R, Järvelä $\mathrm{T}$, Aho $\mathrm{H}$, et al. Validation of the Western Ontario Meniscal Evaluation Tool (WOMET) for patients with a degenerative meniscal tear: a meniscal pathology-specific quality-of-life index. $J$ Bone Joint Surg Am 2012;94:e65.

28. Gronbech Jorgensen M, Andersen S, Ryg J, et al. Novel use of the Nintendo Wii board for measuring isometric lower limb strength: a reproducible and valid method in older adults. PLoS One 2015; 10:e0138660.

29. U. S. Food Drug Administration. What is a serious adverse event? Silver Spring, MD: FDA, 2014. http://www.fda.gov/Safety/ MedWatch/HowToReport/ucm053087.htm (accessed 15 Feb 2017).

30. Conner-Spady BL, Marshall DA, Bohm E, et al. Reliability and validity of the EQ-5D-5L compared to the EQ-5D-3L in patients with osteoarthritis referred for hip and knee replacement. Qual Life Res 2015;24:1775-84.
31. Janssen MF, Pickard AS, Golicki D, et al. Measurement properties of the EQ-5D-5L compared to the EQ-5D-3L across eight patient groups: a multi-country study. Qual Life Res 2013;22:1717-27.

32. Saltin B, Grimby G. Physiological analysis of middle-aged and old former athletes. Comparison with still active athletes of the same ages. Circulation 1968;38:1104-15.

33. Tegner Y, Lysholm J. Rating systems in the evaluation of knee ligament injuries. Clin Orthop Relat Res 1985;198:42-9.

34. Tubach F, Ravaud P, Baron G, et al. Evaluation of clinically relevant states in patient reported outcomes in knee and hip osteoarthritis: the patient acceptable symptom state. Ann Rheum Dis 2005;64:34-7.

35. Ingelsrud LH, Granan LP, Terwee CB, et al. Proportion of patients reporting acceptable symptoms or treatment failure and their associated KOOS values at 6 to 24 months after anterior cruciate ligament reconstruction: a study from the Norwegian knee ligament registry. Am J Sports Med 2015;43:1902-7.

36. Hawker GA, Mian S, Kendzerska T, et al. Measures of adult pain: visual analog scale for pain (VAS Pain), numeric rating scale for pain (NRS pain), McGill Pain Questionnaire (MPQ), Short-Form McGill Pain Questionnaire (SF-MPQ), chronic pain grade scale (CPGS), short form-36 bodily pain scale (SF-36 BPS), and measure of intermittent and constant osteoarthritis pain (ICOAP). Arthritis Care Res 2011;63(S11):S240-S252.

37. Anderson AF, Irrgang JJ, Dunn W, et al. Interobserver reliability of the International Society of Arthroscopy, Knee Surgery and Orthopaedic Sports Medicine (ISAKOS) classification of meniscal tears. Am J Sports Med 2011;39:926-32.

38. Brittberg M, Winalski CS. Evaluation of cartilage injuries and repair. $J$ Bone Joint Surg Am 2003;85-A Suppl 2(-A Suppl 2):58-69.

39. Roos EM, Lohmander LS. The Knee injury and Osteoarthritis Outcome Score (KOOS): from joint injury to osteoarthritis. Health Qual Life Outcomes 2003;1:64.

40. Zou G. A modified poisson regression approach to prospective studies with binary data. Am J Epidemiol 2004;159:702-6. 